\title{
Effect of additional treatment with EXenatide in patients with an Acute Myocardial Infarction (EXAMI): study protocol for a randomized controlled trial
}

\author{
Martijn Scholte', Leo Timmers², Flip JP Bernink', Robert N Denham, Aernout M Beek', Otto Kamp', \\ Michaela Diamant ${ }^{3}$, Anton JG Horrevoets ${ }^{4}$, Hans WM Niessen ${ }^{5}$, Weena JY Chen ${ }^{3}$, Albert C van Rossum ${ }^{1}$, \\ Niels van Royen ${ }^{1}$, Pieter A Doevendans ${ }^{2}$ and Yolande Appelman ${ }^{1}$
}

\begin{abstract}
Background: Myocardial infarction causes irreversible loss of cardiomyocytes and may lead to loss of ventricular function, morbidity and mortality. Infarct size is a major prognostic factor and reduction of infarct size has therefore been an important objective of strategies to improve outcomes. In experimental studies, glucagon-like peptide 1 and exenatide, a long acting glucagon-like peptide 1 receptor agonist, a novel drug introduced for the treatment of type 2 diabetes, reduced infarct size after myocardial infarction by activating pro-survival pathways and by increasing metabolic efficiency.

Methods: The EXAMI trial is a multi-center, prospective, randomized, placebo controlled trial, designed to evaluate clinical outcome of exenatide infusion on top of standard treatment, in patients with an acute myocardial infarction, successfully treated with primary percutaneous coronary intervention. A total of 108 patients will be randomized to exenatide (5 $\mu \mathrm{g}$ bolus in 30 minutes followed by continuous infusion of $20 \mu \mathrm{g} / 24 \mathrm{~h}$ for $72 \mathrm{~h}$ ) or placebo treatment. The primary end point of the study is myocardial infarct size (measured using magnetic resonance imaging with delayed enhancement at 4 months) as a percentage of the area at risk (measured using T2 weighted images at 3-7 days).
\end{abstract}

Discussion: If the current study demonstrates cardioprotective effects, exenatide may constitute a novel therapeutic option to reduce infarct size and preserve cardiac function in adjunction to reperfusion therapy in patients with acute myocardial infarction.

Trial registration: ClinicalTrials.gov: NCT01254123

Keywords: exenatide, glucagon-like peptide 1, myocardial infarction, reperfusion injury

\section{Background}

Myocardial infarction (MI) causes loss of cardiomyocytes and may lead to loss of ventricular function, morbidity and mortality. The impairment of left ventricular function may lead to heart failure and death and has a major impact on the quality of life of the patient. Infarct size is a major prognostic factor after MI[1,2]. Reduction of infarct size

\footnotetext{
* Correspondence: I.timmers@umcutrecht.nl

${ }^{2}$ Deartment of Cardiology, University Medical Center Utrecht, Room number G02.523, Heidelberglaan 100, 3584 CX Utrecht, The Netherlands

Full list of author information is available at the end of the article
}

has therefore been an important objective of strategies to improve clinical outcomes in patients that suffer acute MI.

It was previously demonstrated that early reperfusion of the infarct related coronary artery, usually by primary percutaneous intervention (PCI) limits myocardial necrosis and improves clinical outcome in patients with acute MI[3]. Despite adequate reperfusion, however, most patients still suffer irreversible cardiomyocyte loss. Ironically, part of this loss is induced by the reperfusion itself. Reperfusion induces several biochemical and metabolic changes, which lead to accelerated apoptosis

\section{C) Biomed Central}


of cardiomyocytes[4]. This phenomenon is referred to as reperfusion injury[5].

Multiple strategies have been investigated to reduce reperfusion injury. One of them is glucose-insulin-potassium (GIK) infusion that has been postulated to possess cytoprotective potential and has been investigated in several clinical trials for cardioprotection in patients with MI. Its efficacy is controversial, with some studies reporting a reduction in mortality in patients with acute MI [6-8], whereas most studies demonstrated no beneficial effect[9-11]. GIK infusion is complicated by volume overload, hypoglycemia and hyperkalemia. A promising alternative is Glucagon-Like Peptide (GLP)-1, a gut incretin hormone, which is released by the gut in response to nutrient intake[12]. It facilitates glucose dependent insulin release and also exerts insulinomimetic actions via the GLP-1 receptor, which is also expressed on cardiomyocytes $[13,14]$. GLP-1 has been demonstrated to exert cardioprotective effects in animal models by activating antiapoptotic signaling pathways and by increasing metabolic efficiency[15]. In one small non-randomized clinical study, 21 patients with myocardial infarction and reduced myocardial function were treated with GLP-1 infusion or placebo after successful PCI. GLP-1 treatment resulted in significant improvement of cardiac function[16]. GLP-1 has a short half-life of minutes, being rapidly degraded by dipeptidyl peptidase-4 (DPP-4). Therefore GLP-1 should be administered continuously or administered together with a DPP-4 inhibitor. DPP-4 resistant GLP-1 receptor agonists, such as exendin- 4 and its synthetic variant exenatide, have a longer half-life, making them more attractive for clinical application[17]. Exenatide is currently used as blood-glucose lowering-therapy in patients with type 2 diabetes. In a porcine model of ischemia and reperfusion injury, exenatide reduced myocardial apoptosis and oxidative stress. This resulted in reduced infarct size and preserved cardiac performance[18]. Also, in an isolated rat heart model, exendin-4 provided cardioprotection. This effect was abolished by using a GLP-1 receptor antagonist [19]. These data suggest that exenatide exerts a direct cardioprotective effect via the GLP-1 receptor, rather than an indirect effect via increased insulin levels.

The promising clinical results obtained with GLP-1 and with exenatide in the experimental setting constituted the rationale for the initiation of the EXAMI trial. This randomized placebo controlled multi center study will assess the cardioprotective effects of intravenous exenatide infusion on top of standard treatment in patients with ST elevation myocardial infarction undergoing primary $\mathrm{PCI}$.

\section{Methods}

\section{Overview}

The EXAMI trial is a multi-center, prospective, randomized, placebo controlled clinical trial, designed to evaluate the safety and clinical efficacy of exenatide on top of standard treatment, in patients with an acute MI, successfully treated with PCI (http://www.clinicaltrials.gov; unique identifier NCT01254123). The trial is conducted in two Dutch hospitals: the VU University Medical Center in Amsterdam, and the University Medical Center Utrecht. The study was approved by the institutional ethical committee on human research and will be carried out in compliance with the Helsinki Declaration. To be eligible for participation in the trial, patients have to fit the inclusion and exclusion criteria listed in Table 1. Initially, a pilot study will be performed in the VU University Medical Center Amsterdam, in order to assess the safety and feasibility of exenatide infusion in patients with an acute MI. Forty patients will be randomly assigned to treatment with exenatide or placebo on top of standard treatment. An interim analysis will take place after inclusion of these 40 patients. Also patients that initially receive study medication and are subsequently excluded based on exclusion criteria during coronary angiogram or PCI, will be followed for the registration of potential side effects. If significant negative side effects or significant differences are observed in any of the endpoints in the disadvantage of exenatide, the trial will be put on hold. If exenatide infusion is considered safe, the study will be expanded to a total of 108 patients to assess the cardioprotective effects of exenatide following acute MI.

\section{Patient enrolment and randomization}

Patients with an acute ST elevation myocardial infarction to be treated with primary PCI are potential candidates for the study. Patients that are eligible for participation are asked for informed consent. After informed consent, patients are randomized to exenatide or placebo treatment by using sealed envelopes. Only specially assigned nurses will open the envelopes and will start study medication. All patients are also treated with aspirin, heparin and clopidogrel according to the ESC guidelines. After the start of administration of study medication a coronary angiogram (CAG) and if possible primary PCI will be performed. Patients can be excluded during CAG or primary PCI based on the criteria listed in table 1 . The final CAG will be performed for measurement of Thrombolysis in Myocardial Infarction (TIMI) frame count and TIMI blush grade. During follow up, all patients will be treated with aspirin, clopidogrel, aggressive lipid-lowering treatment, $\mathrm{ACE}$ inhibitor or ARB, and beta-blocker, unless contraindicated. The use of GPIIb/IIIa inhibitors will be left to the discretion of the operator. The flowchart of the study design is shown in Figure 1.

\section{Exenatide treatment protocol}

Byetta injection pens (Amylin Pharmaceuticals, San Diego, CA, USA) containing $1,2 \mathrm{ml}$ exenatide (concentration 0 , 
Table 1 Inclusion and exclusion criteria

\begin{tabular}{|c|c|}
\hline $\begin{array}{l}\text { Inclusion } \\
\text { criteria }\end{array}$ & $>18$ and $<80$ years of age \\
\hline & First myocardial infarction \\
\hline & ST elevation of more than one $\mathrm{mm}$ in at least 2 separate leads on the electrocardiogram (ECG) \\
\hline & Delay between onset of sustained chest pain and $\mathrm{PCl}<6$ hours. \\
\hline Exclusion & Cardiac rhythm is other than normal sinus rhythm. \\
\hline & Patient in Killip class 3 or 4 of heart failure \\
\hline & Cardiogenic shock defined as sustained systolic blood pressure $\leq 80 \mathrm{mmHg}$ despite fluid hydration. \\
\hline & Post cardiac resuscitation \\
\hline & Need for intra aortic balloon counterpulsation therapy \\
\hline & The patient is unable to hold his/her breath for up to 20 seconds due to age or concomitant illness \\
\hline & Former PCl performed \\
\hline & No re-canalization achieved of the occluded coronary artery \\
\hline & Culprit not in segment $1,2,3,6,7,11,12,13$ of the coronary artery \\
\hline & No definite culprit \\
\hline & More than one occluded vessel, or a more than $70 \%$ stenosis by visual assessment in a non-culprit vessel. \\
\hline & TIMI 3 flow in culprit lesion at presentation \\
\hline & Decreased renal function eGFR $<30 \mathrm{~mL} / \mathrm{min} / 1.73 \mathrm{~m}^{2}$ \\
\hline & Any contraindication for MRI \\
\hline & Metal fragments in eye, head, ear, skin or shoulder. \\
\hline & Swann-Ganz catheter. \\
\hline & $\begin{array}{l}\text { Known pre-existing left ventricular dysfunction measured by any technique (ejection fraction }<45 \% \text { prior to current admission for } \\
\text { myocardial infarction) }\end{array}$ \\
\hline & Prior myocardial infarction \\
\hline & Prior coronary artery bypass grafting \\
\hline & Moderate to severe cardiac valve disease \\
\hline & Stroke or transient ischemic attack within the previous 24 hours \\
\hline & Serious known concomitant disease with a life expectancy of less than one year \\
\hline & Follow up impossible \\
\hline & Previous participation in a trial within the previous 30 days \\
\hline & Known type I Diabetes \\
\hline
\end{tabular}

$25 \mathrm{mg} / \mathrm{ml}$; 60 doses of $5 \mu \mathrm{g}$ ) will be obtained. Three doses $(15 \mu \mathrm{g})$ will be diluted in $49 \mathrm{ml}$ saline and $1 \mathrm{ml}$ Human Serum Albumin (Cealb $200 \mathrm{~g} / \mathrm{L}, 10 \mathrm{ml}$ ) to obtain an exenatide concentration of $0.30 \mu \mathrm{g} / \mathrm{ml}$. Infusion will start immediately after informed consent, just prior to PCI. During the first 30 minutes it will be infused intravenously at $33.33 \mathrm{ml} / \mathrm{h}(5 \mu \mathrm{g}$ bolus in 30 minutes $)$, followed by intravenous infusion at $2.78 \mathrm{ml} / \mathrm{h}(20 \mu \mathrm{g} / 24 \mathrm{~h})$ for 72 hours. New solutions will be made every 8 hours. Based on experiences with healthy subjects, this is the maximum well-tolerated intravenous dose.

Nausea is a side effect of exenatide as it slows gastric emptying. It occurs in $40-50 \%$ of the patients, but is usually mild, and severe nausea is reported in $3.5 \%$ of the patients[20]. Nausea will be treated with metoclopramide $20 \mathrm{mg}$ TID. If the nausea does not decrease in response to metoclopramide, the dose of study medication will be lowered to $50 \%$. When serious side effects remain during infusion, study medication will be stopped.

\section{Blood analysis and blood glucose level monitoring}

During hospital admission and follow up visits blood analysis will be performed. An overview of blood analysis during the study is listed in table 2. Blood glucose levels will be monitored every 3 hours during the first 24 hours of study medication treatment, or when hypoglycemia is clinically suspected (Figure 2). When no hypoglycemic (glucose level $<4 \mathrm{mmol} / \mathrm{L}$ ) or hyperglycemic (glucose level $>10 \mathrm{mmol} / \mathrm{L}$ ) episodes occur during the first 24 hours of study medication treatment, blood glucose levels will be monitored 4 times a day until the end of admission. In case of hypoglycemia patients will be treated with $100 \mathrm{ml}$ glucose $50 \%$ infusion and subsequently with glucose $5 \% 1 \mathrm{~L} / 24$ hours. During a 


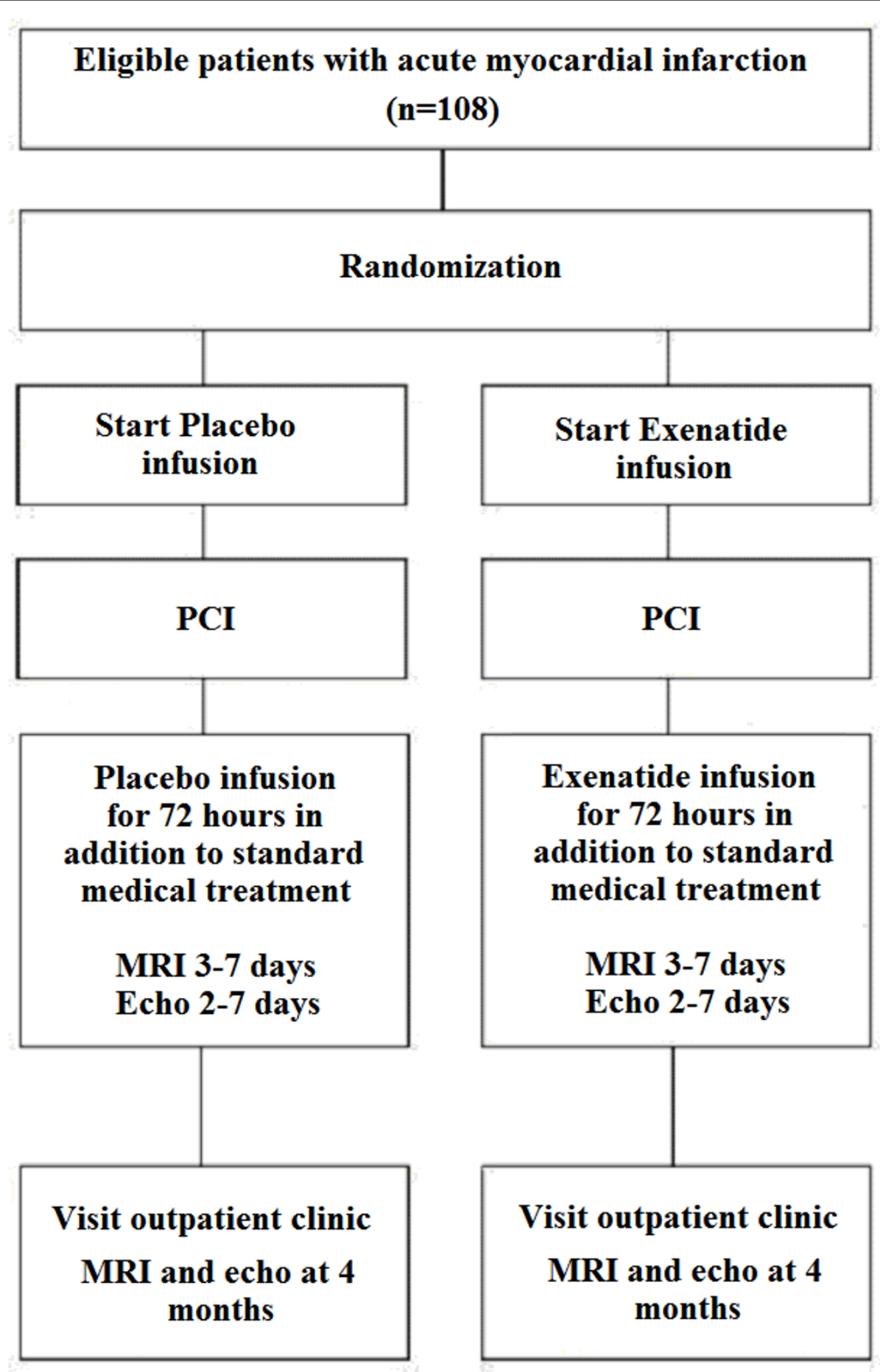

Figure 1 Flowchart of the study design. This figure illustrates the study design. A total of 108 patients will be randomized to Exenatide $(\mathrm{n}=$ 54) or placebo $(n=54)$ infusion. The treatment will be initiated just prior to $\mathrm{PCl}$ and will be continued for 72 hours. Echocardiography and MRI will be performed during hospital admission (within 2-7 days for echo and within 3-7 days for MRI) and at 4 months follow up.

hypoglycemic period blood glucose levels will be assessed every hour until blood glucose levels are $>4$ $\mathrm{mmol} / \mathrm{L}$. During hypoglycemic periods study medication will be continued.
Patients who are hyperglycemic at presentation will be treated with intravenous actrapid as long as necessary to maintain blood glucose levels between 4 and $8 \mathrm{mmol} / \mathrm{L}$. Actrapid will be started at a dose of 1 unit/hour and the 
Table 2 Blood analysis during the study Laboratory measurements on admission:

ESR; CRP; hemoglobin; hematocrit; platelet count; leucocytes; leukocyte differential; sodium; potassium; creatinin; urea; AST; ALT; LDH; bilirubin; CK; CK-MB; troponin; total cholesterol; LDL cholesterol; HDL cholesterol; plasma glucose; HbA1c; NT-proBNP; albumin; homocysteine; Lipoproteine(a); Insulin; C-peptide; Free Fatty Acids; APTT; INR;

Routine laboratory measurements (every CRP, hemoglobin; hematocrit; platelet count; leucocytes; sodium; potassium; creatinin; exenatide morning at 9:00) serum level (during duration of study medication), insulin, C-peptide.

Other laboratory measurements

On the fourth day after admission a triglyceride level and a blood glucose level will be obtained in the fasting state. Exenatide serum levels, insulin and C-peptide will be measured $4 \mathrm{~h}$ after initiation of treatment.

Laboratory measurements at follow up visit at 1 month and 4 months

(RP, hemoglobin; hematocrit; platelet count; leucocytes; sodium; potassium; creatinin; AST; ALT; LDH; total cholesterol; LDL cholesterol; HDL cholesterol; blood glucose level; HbA1c; NTproBNP; albumine; Lipoproteine (a)

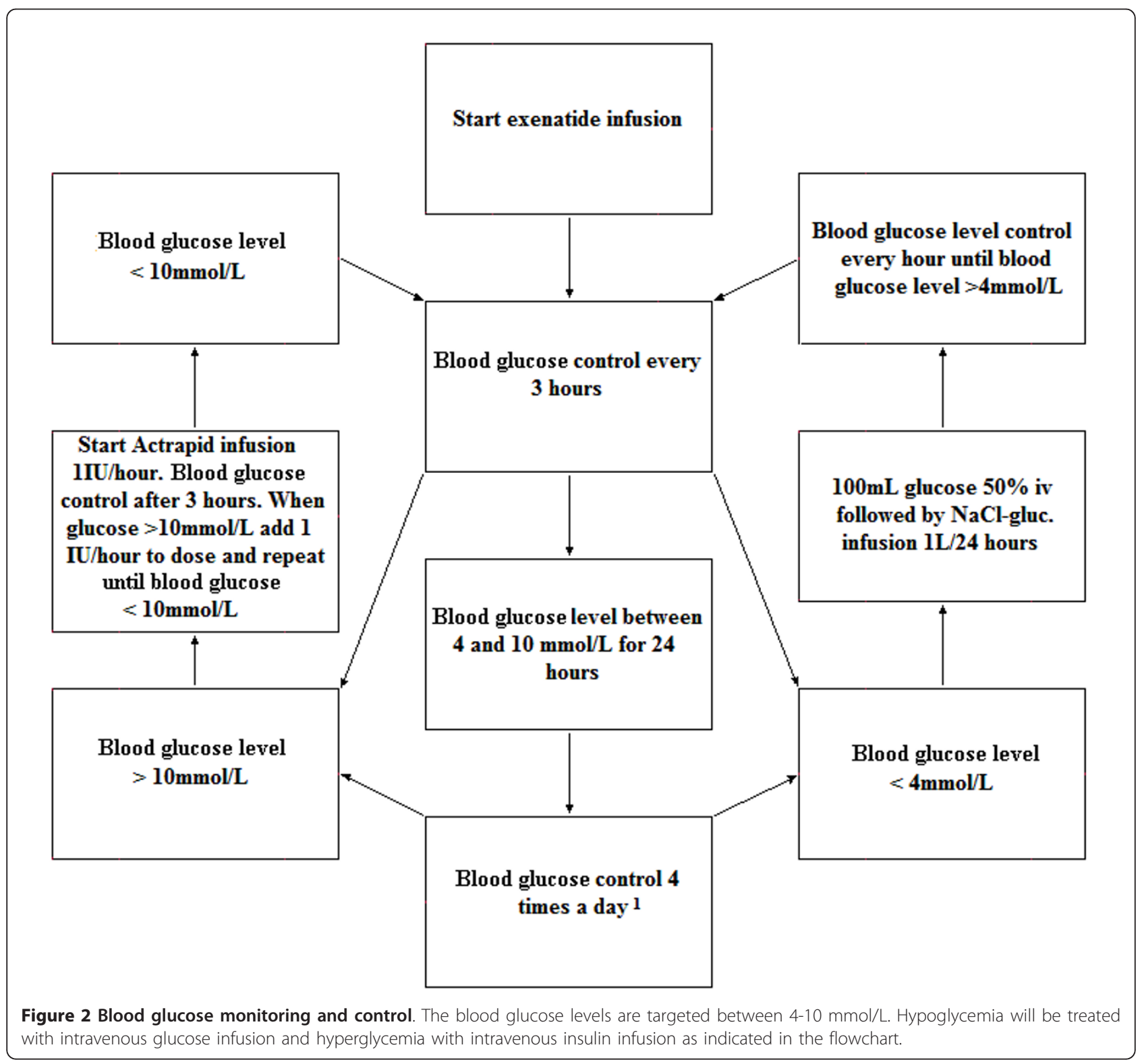


dose will be adjusted according to blood glucose levels that will be checked every three hours.

Mild to moderate hypoglycemia is expected in approximately $5 \%$ of the patients, severe hypoglycemia is not expected[20].

\section{Magnetic Resonance Imaging (MRI)}

Three to 7 days and 4 months after primary PCI a cardiac MRI with delayed contrast enhancement (DCE) will be performed to assess left ventricular function and infarction size. The first MRI is performed to visualize myocardial edema, i.e. the area at risk. The second MRI is preformed to measure myocardial fibrosis, i.e. infarct area. Doing so, infarct size can be measured as a percentage of the area at risk. Patients are studied on a clinical 1, 5 Tesla scanner with a cardiac phased array receiver surface coil placed on the thorax.

ECG-gated cine MR images are obtained during repeated breath-holds in the 3 standard long axis views (4, 3- and 2-chamber view). Additional short axis slices are acquired covering the entire left ventricle to examine regional and global left ventricular function. T2-weighted imaging will be acquired in long and short axis views and will be used to estimate the area at risk[21-23]. During intravenous injection of $0,10 \mathrm{mmol} / \mathrm{kg}$ gadoliniumdiethyltriaminepenta-acetic acid, first-pass perfusion imaging is performed. Delayed contrast enhanced images are acquired 10 minutes post-contrast to identify the location and extent of myocardial infarction.

All images are stored on a database where it will be forwarded for later blinded analysis. The MRI data are analyzed using a software package (Mass, Medis, Leiden, the Netherlands). On the short axis cine slices, the endocardial and epicardial borders are outlined manually in end-diastolic and end-systolic images, excluding trabeculae and papillary muscles. Assessment of global left ventricular function is obtained by calculating left ventricular volumes, mass, and ejection fraction using the summation of slice method multiplied by slice distance. For analysis of segmental myocardial function, each short axis slice is divided in 12 equiangular segments, starting at the posterior septal insertion of the right ventricle. Segmental wall thickening is expressed in absolute values (end-diastolic wall thickness subtracted from end-systolic wall thickness [in millimeters]) and relative values (absolute wall thickening divided by end-diastolic wall thickness [\%]). Areas of hyperenhancement are outlined, including central dark zones of microvascular obstruction, allowing the calculation of total infarct size by summation of all slice volumes of hyperenhancement.

\section{Echocardiography}

Two to 7 days and 4 months after primary PCI, transthoracic echocardiography will be performed to measure global and regional cardiac function, using the iE33 ultrasound system (Philips Medical Systems, Andover, MA, USA) equipped with a S5-2 phased array ( 1 to $5 \mathrm{MHz}$ broadband) transducer (Philips Medical Systems, Andover, MA, USA). A systematic imaging protocol will be performed, consisting of parasternal long-axis, short-axis and 4-, 2-, and 3-chamber apical views, closely following current guidelines. Global function of the left ventricle will be assessed with biplane Simpson's method. Global right ventricular function will be measured with the functional parameter tricuspid annular plane systolic excursion (TAPSE). Mitral, tricuspid, pulmonary and aortic valve will be assessed with color Doppler, measuring filling times, E- and A-top of the mitral valve and valvular function. In addition, the diastolic left ventricular function will be assessed with coded tissue Doppler imaging measuring peak tissue velocity during early diastole (E') at the basal left ventricular septum and lateral wall. Finally, using the same ultrasound system equipped with a X3-1 matrix-array transducer (Philips Medical Systems, Andover, MA, USA) a complete real-time 3-dimensional echo dataset will be obtained for blinded off-line analysis of global and regional left ventricular function.

\section{Follow up}

All patients visit our outpatient clinic at 6 weeks and at 4 months after primary PCI. The visits consist of clinical evaluation, blood analysis, and 12-lead electrocardiogram. Major cardiovascular events (death, myocardial re-infarction, coronary artery bypass grafting, repeat PCI) will be documented. Furthermore, cardiac arrhythmias, heart failure, repeat coronary angiography, stroke, and hospital admission) are documented. At 4 months a second MRI and echocardiography will be performed to assess cardiac function and infarct size.

\section{End points}

The primary endpoint of the study is myocardial infarct size as a percentage of the area at risk (i.e. the perfusion territory of the infarct related coronary artery) as measured by MRI. The area at risk is measured 3-7 days after primary PCI and myocardial infarct size is measured 4 months after primary PCI. All secondary endpoints are indicated in table 3.

\section{Sample Size}

Myocardial infarct size as a percentage of the area at risk will be the primary endpoint of the study. With a power of 0.9 and an alpha error of 0.05 , and with an anticipated $15 \%$ reduction in myocardial infarct size in the treatment arm considered clinically relevant, 48 patients per treatment arm have to be enrolled. To allow for dropout of $10 \%$ (based on previous experience), a total of 108 patients will be enrolled. 
Table 3 Endpoints

\begin{tabular}{|c|c|}
\hline Primary endpoint & $\begin{array}{l}\text { Infarct size measured as the final infarct size on delayed contrast enhancement (DCE) MRI at } 4 \text { months as a percentage of the } \\
\text { area at risk on T2 weighted MRI at 3-7 days. }\end{array}$ \\
\hline \multirow{10}{*}{$\begin{array}{l}\text { Secondary } \\
\text { endpoints }\end{array}$} & Myocardial salvage index and final infarct size measured by MRI \\
\hline & $\begin{array}{l}\text { Regional cardiac function based on echocardiographic and MRI segmental analysis at 2-7 days and at } 4 \text { months (for echo) and } \\
\text { at 3-7 days and at } 4 \text { months (for MRI). }\end{array}$ \\
\hline & Global cardiac functional parameters measured by echocardiography and MRI \\
\hline & Myocardial infarct size as measured by serum CK-MB release during the first 72 hours after PCl. \\
\hline & Microvascular obstruction measured by DCE MRI 3-7 days after PCI. \\
\hline & Blood pressure and heart rate at 1 day, 7 days and at 4 months \\
\hline & $\begin{array}{l}\text { The occurrence within } 4 \text { months of a Major Adverse Cardiac Event (MACE) defined as cardiac death, myocardial infarction, } \\
\text { coronary bypass grafting, or a repeat PCI. }\end{array}$ \\
\hline & Repeat PCI in the infarct related artery during 4 months follow up. \\
\hline & Plasma glucose levels during the first 72 hours. \\
\hline & Side effects of exenatide \\
\hline
\end{tabular}

\section{Statistical Analysis}

The study results will be evaluated based on intention to treat analysis. Depending on the distribution of continuous outcomes, independent samples t-test or MannWhitney $U$ test will be used as appropriate. Binary end points will be compared using Fisher exact probability test. For survival and clinical events, Kaplan Meijer curves will be displayed and comparison of the curves will be done using log rank testing based on a proportional hazards model.

\section{Conclusion and implications}

The EXAMI study, as outlined above, is a randomized clinical trial to investigate the cardioprotective properties of exenatide in patients with acute MI undergoing primary PCI, by means of measuring final infarct size compared to area at risk as measured with MRI. If reduction in infarct size is accomplished, exenatide may constitute a valuable therapeutic auxiliary for reperfusion therapy in patients with acute MI.

\section{Trial status}

Currently including patients

\begin{abstract}
Author details
${ }^{1}$ Department of Cardiology, VU University Medical Center, De Boelelaan 11171081 HV Amsterdam, The Netherlands. ${ }^{2}$ Deartment of Cardiology, University Medical Center Utrecht, Room number G02.523, Heidelberglaan 100, 3584 CX Utrecht, The Netherlands. ${ }^{3}$ Department of Endocrinology and Diabetes Centre, VU University Medical Centre, PO Box 7057, 1007 MB Amsterdam, The Netherlands. ${ }^{4}$ Department of Molecular Cell Biology and Immunology, VU University Medical Center, Van der Boechorststraat 7, 1081 BT Amsterdam, The Netherlands. ${ }^{5}$ Department of Pathology, VU University Medical Cente, rPO Box 7057, 1007 MB, Amsterdam, The Netherlands.
\end{abstract}

\section{Authors' contributions}

MS made contributions to conception and design and of the trial, data acquisition and has been involved in drafting the manuscript. LT contributed to conception and design of the trial and drafted the manuscript. FB, RD and WC contributed to data acquisition and critically revised the manuscript for important intellectual content. AB contributed to trial design, designed the MRI protocols and critically revised the manuscript for important intellectual content. OK contributed to trial design, designed the echocardiography protocols and critically revised the manuscript for important intellectual content. MD, AH, HN, AR, NR, PD and YA contributed to conception and design, and critically revised the manuscript for important intellectual content. All authors have given final approval of the version of the manuscript to be published.

\section{Competing interests}

The authors declare that they have no competing interests.

Received: 1 August 2011 Accepted: 8 November 2011 Published: 8 November 2011

\section{References}

1. Gibbons RJ, Valeti US, Araoz PA, Jaffe AS: The quantification of infarct size. J Am Coll Cardiol 2004, 44:1533-1542.

2. Burns RJ, Gibbons RJ, Yi Q, Roberts RS, Miller TD, Schaer GL, Anderson JL, Yusuf S: The relationships of left ventricular ejection fraction, end-systolic volume index and infarct size to six-month mortality after hospital discharge following myocardial infarction treated by thrombolysis. J Am Coll Cardiol 2002, 39:30-36.

3. O'Neill W, Timmis GC, Bourdillon PD, Lai P, Ganghadarhan V, Walton J Jr, Ramos R, Laufer N, Gordon S, Schork MA, et al: A prospective randomized clinical trial of intracoronary streptokinase versus coronary angioplasty for acute myocardial infarction. N Engl J Med 1986, 314:812-818.

4. Eefting F, Rensing B, Wigman J, Pannekoek WJ, Liu WM, Cramer MJ, Lips DJ, Doevendans PA: Role of apoptosis in reperfusion injury. Cardiovasc Res 2004, 61:414-426.

5. Yellon DM, Hausenloy DJ: Myocardial reperfusion injury. N Engl J Med 2007, 357:1121-1135.

6. Fath-Ordoubadi F, Beatt KJ: Glucose-insulin-potassium therapy for treatment of acute myocardial infarction: an overview of randomized placebo-controlled trials. Circulation 1997, 96:1152-1156.

7. van der Horst IC, Zijlstra F, van 't Hof AW, Doggen CJ, de Boer MJ, Suryapranata H, Hoorntje JC, Dambrink JH, Gans RO, Bilo HJ: Glucoseinsulin-potassium infusion inpatients treated with primary angioplasty for acute myocardial infarction: the glucose-insulin-potassium study: a randomized trial. J Am Coll Cardiol 2003, 42:784-791.

8. Malmberg K, Ryden L, Efendic S, Herlitz J, Nicol P, Waldenstrom A, Wedel H, Welin L: Randomized trial of insulin-glucose infusion followed by subcutaneous insulin treatment in diabetic patients with acute 
myocardial infarction (DIGAMI study): effects on mortality at 1 year. $J$ Am Coll Cardiol 1995, 26:57-65.

9. Diaz R, Paolasso EA, Piegas LS, Tajer CD, Moreno MG, Corvalan R, Isea JE, Romero G: Metabolic modulation of acute myocardial infarction. The ECLA (Estudios Cardiologicos Latinoamerica) Collaborative Group. Circulation 1998, 98:2227-2234.

10. Ceremuzynski L, Budaj A, Czepiel A, Burzykowski T, Achremczyk P, SmielakKorombel W, Maciejewicz J, Dziubinska J, Nartowicz E, Kawka-Urbanek T, et al: Low-dose glucose-insulin-potassium is ineffective in acute myocardial infarction: results of a randomized multicenter Pol-GIK trial. Cardiovasc Drugs Ther 1999, 13:191-200.

11. Mehta SR, Yusuf S, Diaz R, Zhu J, Pais P, Xavier D, Paolasso E, Ahmed R, Xie $C$, Kazmi $K$, et al: Effect of glucose-insulin-potassium infusion on mortality in patients with acute ST-segment elevation myocardial infarction: the CREATE-ECLA randomized controlled trial. Jama 2005, 293:437-446.

12. Goke R, Fehmann HC, Linn T, Schmidt H, Krause M, Eng J, Goke B: Exendin4 is a high potency agonist and truncated exendin-(9-39)-amide an antagonist at the glucagon-like peptide 1-(7-36)-amide receptor of insulin-secreting beta-cells. J Biol Chem 1993, 268:19650-19655.

13. Wei Y, Mojsov S: Tissue-specific expression of the human receptor for glucagon-like peptide-l: brain, heart and pancreatic forms have the same deduced amino acid sequences. FEBS Lett 1995, 358:219-224.

14. Vila Petroff MG, Egan JM, Wang X, Sollott SJ: Glucagon-like peptide-1 increases CAMP but fails to augment contraction in adult rat cardiac myocytes. Circ Res 2001, 89:445-452.

15. Bose AK, Mocanu MM, Carr RD, Brand CL, Yellon DM: Glucagon-like peptide 1 can directly protect the heart against ischemia/reperfusion injury. Diabetes 2005, 54:146-151.

16. Nikolaidis LA, Mankad S, Sokos GG, Miske G, Shah A, Elahi D, Shannon RP: Effects of glucagon-like peptide- 1 in patients with acute myocardial infarction and left ventricular dysfunction after successful reperfusion. Circulation 2004, 109:962-965.

17. Davidson MB, Bate G, Kirkpatrick P: Exenatide. Nat Rev Drug Discov 2005, 4:713-714.

18. Timmers $L$, Henriques JP, de Kleijn DP, Devries $J H$, Kemperman $H$, Steendijk P, Verlaan CW, Kerver M, Piek JJ, Doevendans PA, et al: Exenatide reduces infarct size and improves cardiac function in a porcine model of ischemia and reperfusion injury. J Am Coll Cardiol 2009, 53:501-510.

19. Sonne DP, Engstrom T, Treiman M: Protective effects of GLP-1 analogues exendin-4 and GLP-1(9-36) amide against ischemia-reperfusion injury in rat heart. Regul Pept 2008, 146:243-249.

20. King $A B$, Wolfe $G$, Healy $S$ : Clinical observations of exenatide treatment. Diabetes Care 2006, 29:1984.

21. Aletras AH, Tilak GS, Natanzon A, Hsu LY, Gonzalez FM, Hoyt RF Jr, Arai AE: Retrospective determination of the area at risk for reperfused acute myocardial infarction with T2-weighted cardiac magnetic resonance imaging: histopathological and displacement encoding with stimulated echoes (DENSE) functional validations. Circulation 2006, 113:1865-1870.

22. Carlsson M, Ubachs JF, Hedstrom E, Heiberg E, Jovinge S, Arheden H: Myocardium at risk after acute infarction in humans on cardiac magnetic resonance: quantitative assessment during follow-up and validation with single-photon emission computed tomography. JACC Cardiovasc Imaging 2009, 2:569-576.

23. Wright J, Adriaenssens T, Dymarkowski S, Desmet W, Bogaert J: Quantification of myocardial area at risk with T2-weighted CMR: comparison with contrast-enhanced CMR and coronary angiography. JACC Cardiovasc Imaging 2009, 2:825-831.

doi:10.1186/1745-6215-12-240

Cite this article as: Scholte et al.: Effect of additional treatment with

EXenatide in patients with an Acute Myocardial Infarction (EXAMI):

study protocol for a randomized controlled trial. Trials 2011 12:240.

\section{Submit your next manuscript to BioMed Central and take full advantage of:}

- Convenient online submission

- Thorough peer review

- No space constraints or color figure charges

- Immediate publication on acceptance

- Inclusion in PubMed, CAS, Scopus and Google Scholar

- Research which is freely available for redistribution 